\section{Transformation Mechanism and Kinetics for the Pressure-Induced Phase Transition in Shocked CdS}

\author{
M.D. Knudson ${ }^{1}$, Y.M. Gupta ${ }^{1}$, and A.B. Kunz ${ }^{2}$ \\ ${ }^{1}$ Institute for Shock Physics and Department of Physics, Washington State University, Pullman, WA 99163 \\ ${ }^{2}$ Department of Electrical Engineering, Michigan Tech. University, Houghton, MI 49931 \\ *Current address Sandia National Laboratories, Albuquerque, NM 87185
}

RECEIVED

JUL 131999

OSTI

\begin{abstract}
The pressure-induced phase transition in CdS was investigated using picosecond timeresolved electronic spectroscopy in plate impact shock wave experiments. Real-time changes in the electronic spectra were observed, with $100 \mathrm{ps}$ time resolution, in single crystals of CdS shocked along the $c$ and $a$ axes to peak stresses between 35 and $90 \mathrm{kbar}$ (above the phase transition stress of approximately $30 \mathrm{kbar}$ measured in continuum studies). When shocked to stresses above approximately $50 \mathrm{kbar}$ along the crystal $c$ axis and 60 to $70 \mathrm{kbar}$ along the crystal $a$ axis, the crystals undergo a very rapid change in electronic structure, indicating that significant structural changes occur within the first $100 \mathrm{ps}$. These results, along with previous ns continuum measurements, make a strong case for a metastable state during the phase transition in shocked CdS. Ab-initio periodic Hartree-Fock calculations (with DFT correlation corrections) were employed to examine the compression of CdS and to determine a possible lattice structure for the proposed metastable structure. These results, along with details of the transformation kinetics and orientational dependence, will be discussed. Work supported by ONR.
\end{abstract}

\section{INTRODUCTION}

Plate impact, shock wave experiments provide a unique method to investigate the time-dependent mechanisms and the kinetics associated with pressure-induced phenomena, such as chemical reactions and phase transformations. The very rapid and well defined loading conditions associated with plate-impact experiments permit real-time examination of the shock-induced changes. Further, the ability to propagate the shock wave along various crystallographic directions provides the means to perform careful analysis of the stress and orientational dependence.

Recently, an experimental method has been developed to observe real-time changes in the absorption/transmission of materials, with 100 or 200 ps resolution, in single-event, plate impact shock experiments [1-4]. These data can provide useful information regarding the material under investigation. In particular, the dependence of the absorption edge on photon energy can distinguish between direct and indirect electronic transitions, and can provide an estimate of the band-gap energy of the material [5]. Along with ab-initio techniques to calculate the electronic structure of a crystalline system, this electronic information can be used to gain insight regarding the crystal structure.

As described in Ref. [1,2,4] the wurtzite-torocksalt phase transition in cadmium sulfide (CdS) is well suited to investigation through the use of fast electronic spectroscopy; the wurtzite and rocksalt phases exhibit a direct and indirect band gap with band gap energies of 2.5 and $1.5-1.7 \mathrm{eV}$, respectively [6-8]. The intent of this work was to use picosecond electronic spectroscopy and $a b$ initio methods to examine the real-time structural changes that occur in the initial stages of the shockinduced wurtzite-to-rocksalt phase transition in single crystal CdS. 


\section{DISCLAIMER}

This report was prepared as an account of work sponsored by an agency of the United States Government. Neither the United States Government nor any agency thereof, nor any of their employees, make any warranty, express or implied, or assumes any legal liability or responsibility for the accuracy, completeness, or usefuiness of any information, apparatus, product, or process disclosed, or represents that its use would not infringe privately owned rights. Reference herein to any specific commercial product, process, or service by trade name, trademark, manufacturer, or otherwise does not necessarily constitute or imply its endorsement, recommendation, or favoring by the United States Government or any agency thereof. The views and opinions of authors expressed herein do not necessarily state or reflect those of the United States Government or any agency thereof. 


\section{DISCLAIMER}

Portions of this document may be illegible in electronic image products. Images are produced from the best available original document. 
Table 1. Summary of impact experiments. The fraction transformed was determined relative to experiments $A 7$ and $C 8$ for the $a$ and $c$ axis experiments, respectively. For the experiments marked with an asterisk there was no significant changes in the transmission observed within the experimental duration (12.7-25.4 ns). For the experiments marked with a cross there was significant changes observed, however, the observed kinetics were too slow to obtain information concerning the band gap and the relative fraction transformed through the analysis described here.

\begin{tabular}{|c|c|c|c|c|c|c|c|}
\hline $\begin{array}{c}\text { Experiment } \\
\text { number }\end{array}$ & $\begin{array}{l}\text { Impactor } \\
\text { material }\end{array}$ & $\begin{array}{c}\text { Buffer } \\
\text { material }\end{array}$ & $\begin{array}{c}\text { Sample } \\
\text { orientation }\end{array}$ & $\begin{array}{l}\text { Projectile } \\
\text { velocity } \\
(\mathrm{mm} / \mu \mathrm{s})\end{array}$ & $\begin{array}{c}\text { Calculated } \\
\text { instantaneous } \\
\text { stress (kbar) }\end{array}$ & $\begin{array}{c}\text { Band-gap } \\
\text { energy } \\
\text { (eV) }\end{array}$ & $\begin{array}{l}\text { Relative } \\
\text { fraction } \\
\text { transformed }\end{array}$ \\
\hline C1 (97-524) & fused silica & sapphire & $c$ axis & 0.604 & 35.3 & $\ldots \ldots$ & -----* \\
\hline C2 $(97-525)$ & fused silica & sapphire & $c$ axis & 0.599 & 35.1 & -.---* & ----* \\
\hline$C 3(97-542)$ & fused silica & $\alpha$ quartz & $c$ axis & 0.605 & 46.4 & $\ldots \ldots *$ & -...* \\
\hline C4 (97-562) & fused silica & $\alpha$ quartz & $c$ axis & 0.603 & 46.3 & $\ldots \ldots *$ & --.--* \\
\hline C5 (98-511) & $\alpha$ quartz & $\alpha$ quartz & $c$ axis & 0.551 & 53.4 & 1.51 & 0.38 \\
\hline C6 (97-565) & $\alpha$ quartz & $\alpha$ quartz & $c$ axis & 0.603 & 58.6 & 1.53 & 0.46 \\
\hline C7 $(98-506)$ & sapphire & sapphire & $c$ axis & 0.495 & 71.6 & 1.52 & 0.74 \\
\hline$C 8(97-546)$ & sapphire & sapphire & $c$ axis & 0.607 & 87.8 & 1.52 & 1.0 \\
\hline A1 $(97-566)$ & fused silica & sapphire & $a$ axis & 0.602 & 34.5 & - - * * & - - \\
\hline A2 (97-541) & fused silica & $\alpha$ quartz & $a$ axis & 0.594 & 45.1 & $--^{\dagger}$ & $--_{-}^{\dagger}$ \\
\hline A3 (97-549) & fused silica & $\alpha$ quartz & $a$ axis & 0.605 & 45.9 & $\ldots+. . .{ }^{\dagger}$ & $-\cdots^{\dagger}$ \\
\hline A4 $(97-550)$ & $\alpha$ quartz & $\alpha$ quartz & $a$ axis & 0.605 & 58.3 & $-\cdots-^{\dagger}$ & $--_{--{ }^{\dagger}}^{\dagger}$ \\
\hline A5 $(97-567)$ & sapphire & sapphire & $a$ axis & 0.496 & 71.0 & 1.50 & 0.63 \\
\hline A6 $(97-521)$ & sapphire & sapphire & $a$ axis & 0.601 & 86.4 & 1.51 & 0.88 \\
\hline A7 (97-543) & sapphire & sapphire & $a$ axis & 0.604 & 86.9 & 1.49 & 1.0 \\
\hline
\end{tabular}

\section{EXPERIMENTAL METHODS AND RESULTS}

The experimental methods are briefly described here. A comprehensive discussion can be found elsewhere [1-4]. All of the experiments were performed using either $c$ or $a$ axis, high purity, 5mm-square by $250-\mu \mathrm{m}$-thick CdS single crystals.

Shock waves were generated by impact of a transparent impactor, mounted on a projectile accelerated by a light gas gun [9], onto the target assembly. The latter consisted of two optically transparent buffer windows backed by the sample. Piezoelectric trigger pins were placed behind the front buffer window to provide both a trigger. pulse from the shock front and a timing fiducial.

Fluorescence from a Nd:YAG pumped dye solution was used as the light source for the experiments. Two flat mirrors, located on the projectile, directed the light through the sample where it was collected into an optical fiber bundle; this arrangement provided single pass transmission through the sample. One fiber directed the signal to a fast photodiode used as a timing diagnostic. The second fiber delivered the transmitted signal to the picosecond detection system (spectrometer/streak camera/CCD), which provided intensity data as a function of both wavelength and time. Details concerning the detection system and synchronization in the experiments can be found elsewhere [1-4].

A total of 15 experiments with 100 or 200 ps time resolution were performed on $a$ and $c$ axis CdS single crystals. Table I lists the pertinent parameters for each experiment. The calculated instantaneous or elastic stresses for these experiments ranged between 35 and $90 \mathrm{kbar}$ in the CdS crystal. It is noted that over this stress range $\mathrm{CdS}$ does not respond as a simple elastic material in the longer time (hundreds of ns) continuum studies; shock compression along the crystal $c$ axis results in a structural phase transition at a stress value of 32.5 kbar [10], and shock compression along the crystal $a$ axis results in an elastic-plastic transition and a structural phase transition at stresses of 25.5 and $29.2 \mathrm{kbar}$ [11], respectively. The elastic stress values are used here for ease of discussion and to permit consistent comparison between experiments.

The results of the absorption/transmission measurements showed definite stress and orientational dependence. Fig.1 displays the results obtained from a typical high stress experiment; an $a$ axis crystal shocked to $71.0 \mathrm{kbar}$ (experiment A5). Fig. 1(a) shows the absorbance as a function of both wavelength and time. The time dependence of the absorbance is most easily seen in Fig. 1(b), which 

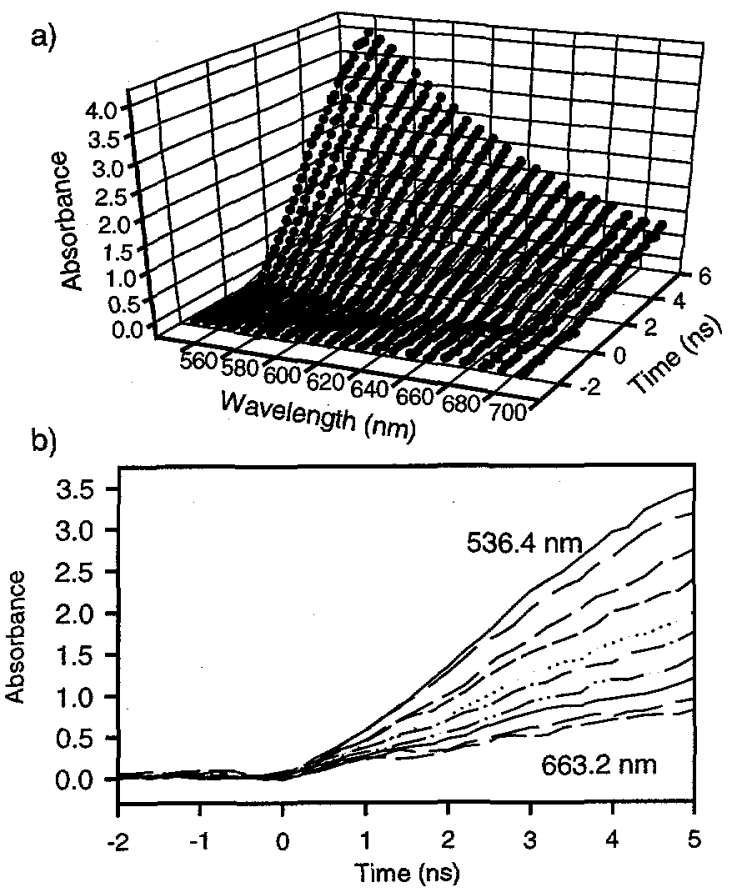

FIGURE 1. Results for an experiment on $a$ axis, CdS shocked to 71.0 kbar. $t=0$ corresponds to the arrival of the shock wave at the CdS sample.

shows absorbance as a function of time for several different wavelengths. In these high stress experiments (experiments $\mathrm{C} 5-8$ and A5-7) it was found that the absorbance at a given wavelength increased linearly with time, with a slope that increased with decreasing wavelength. Further, the onset of the absorbance increase corresponded with the arrival of the shock front at the CdS sample, as determined by the photodiode diagnostic and the calculated traversal time of the shock wave through the buffer window $[1,2]$.

As described in Ref. $[1,2,4]$ the linear increase in absorbance with time observed in these high stress experiments suggests that the absorption coefficient of the material behind the shock front, though wavelength dependent, is time independent; the linear increase reflects the propagation of the shock wave through the CdS sample. Given the shock velocity through the sample $[10,11]$, the absorption coefficient of the material behind the shock front in the high stress experiments was determined from the slope of the absorbance increase [1,2]. The absorption coefficients determined in this way for the various high stress experiments are shown in

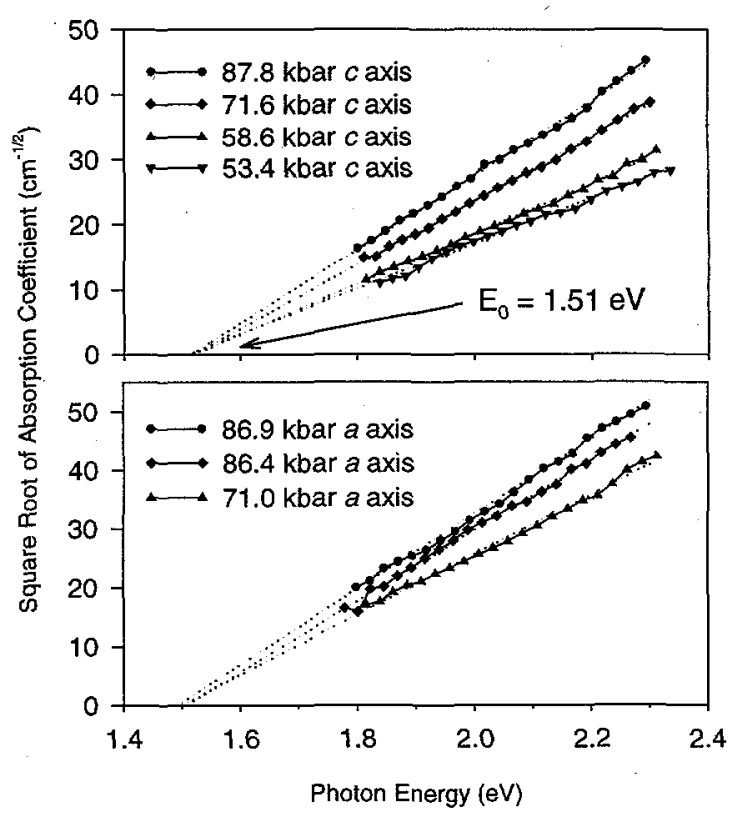

FIGURE 2. Square root of the absorption coefficient as a function of photon energy for the $c$ and $a$ axis experiments.

Fig. 2, displayed as the square root of the absorption coefficient as a function of photon energy.

The linearity of the data suggests that the absorption coefficient observed behind the shock front in the high stress experiments is due to an indirect electronic transition with a band-gap energy of $1.51 \pm 0.02 \mathrm{eV} \mathrm{[5],} \mathrm{comparable} \mathrm{to} \mathrm{that} \mathrm{of} \mathrm{the}$ high pressure rocksalt phase $[7,8]$. This is consistent with the interpretation of a very rapid $(<100 \mathrm{ps})$ structural transition $[1,4]$. However, as described in Ref. $[2,4]$, despite the fact that band-gap energy of the material behind the shock front is comparable to that of the high pressure rocksalt phase of CdS $[7,8]$, we do not believe that the material directly behind the shock front has transformed to the final rocksalt phase. Rather, in accordance with the conclusions of Tang and Gupta [10] and Sharma and Gupta [11], whose continuum experiments on CdS suggest that the transformation proceeds by a two-step process, we propose that the state of the material directly behind the shock front is a metastable structure of CdS.

The slope of the curves shown in Fig. 2 increase with increasing instantaneous stress. This suggests that the fraction of the crystal that undergoes the structural transition increases with instantaneous 

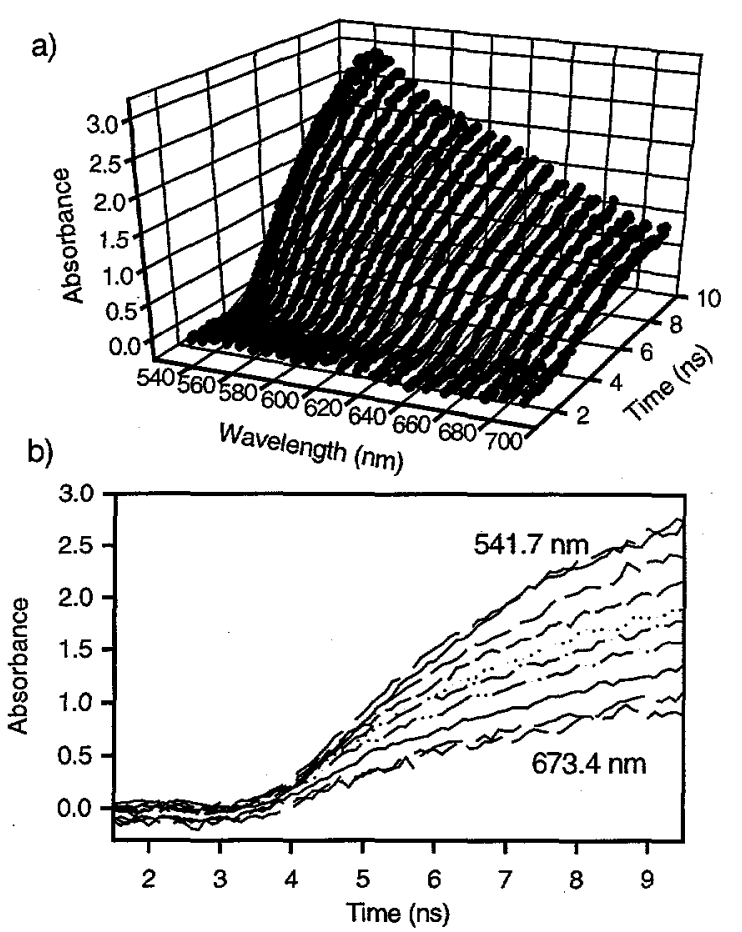

FIGURE 3. Results for an experiment on $a$ axis, CdS shocked to $45.9 \mathrm{kbar} . t=0$ corresponds to the arrival of the shock wave at the CdS sample.

stress $[1,4]$. The relative fraction of the crystal that transformed to the new phase in each experiment can be determined by comparing the relative slope of the curves in Fig. 2 [4]. The fraction transformed in the high stress experiments relative to experiments $\mathrm{A} 7$ and $\mathrm{C} 8$ for the $a$ and $c$ axis experiments, respectively, are shown in Table I.

Fig. 3 displays the results obtained for a typical experiment at slightly lower instantaneous stress; an $a$ axis crystal shocked to $45.9 \mathrm{kbar}$ (experiment A3). In contrast to the high stress experiments, these experiments (experiments A2-4) exhibited a non-linear increase in absorbance, with a more gradual onset in the absorbance increase that occurred several ns after the arrival of the shock wave at the CdS sample. These results suggest that transformation kinetics on the order of a few to tens of ns are involved. This information can provide insight into the nucleation and growth of the new phase and will be described in a future publication.

The lowest stress experiments (experiments $\mathrm{C} 1-4$ and A1) showed no significant change in the transmission within the time interval of the experiments (12.7 to $25.4 \mathrm{~ns}$ ). This is despite the fact that these stresses are above the phase transition stresses of 32.5 and $29.2 \mathrm{kbar}$ reported in continuum studies for shock compression along the $c$ and $a$ axes, respectively $[10,11]$. These results suggest that for crystals shocked to instantaneous stress below approximately $50 \mathrm{kbar}$ and $40 \mathrm{kbar}$ along the $c$ and $a$ axis, respectively, the early time response (12.7 to $25.4 \mathrm{~ns}$ ) of the crystal is similar to the response of the crystal shocked to stresses below the phase transition stress (i.e. that of the compressed wurtzite phase).

Based upon the experimental results the following picture emerges concerning the early stages of the transition. When shocked to instantaneous stresses slightly above the phase transition stress, no significant structural transformation of the CdS crystal occurs within the initial 12.7 to $25.4 \mathrm{~ns}$ after the arrival of the shock wave at the sample; the crystal responds as compressed wurtzite. However, continuum measurements show that transformation to the high pressure rocksalt phase occurs within the first 100 to $200 \mathrm{~ns}[10,11]$. As the instantaneous stress increases, the kinetics associated with structural transformation likewise increases; in an intermediate stress range for $a$ axis compression a few to tens of ns are required to reach a constant state in the transformation to the metastable structure. When shocked to sufficiently high stress, CdS undergoes a very rapid transformation $(<100$ ps) from the initial wurtzite structure to a metastable structure that exhibits an indirect band gap of approximately $1.51 \pm 0.02 \mathrm{eV}$, comparable to that of the final rocksalt phase.

\section{AB-INITIO CALCULATIONS}

In an attempt to link the electronic changes observed in the experimental study with structural changes in the crystal, ab-initio total energy and electronic structure calculations were performed using the CRYSTAL95 code [12]. The model chemistry consisted of a periodic Hartree-Fock selfconsistent-field (SCF) procedure, with a Lee-YangParr density-functional correlation potential [13], included within the SCF cycle, to account for electron correlation. Before examining possible lattice structures for the proposed metastable state, computational results were carefully compared with 


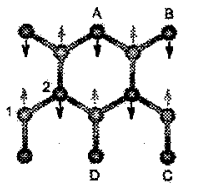

Wurtzite

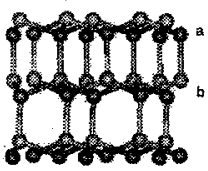

FIGURE 4. The wurtzite, fct, and rocksalt phases of CdS. The top half shows a view down the crystal $c$ axis, the bottom half shows a view orthogonal to that. The dark atoms are Cd.

experiments to ensure that the computational method and basis sets described the system accurately. The comparison between computation and experiment, along with more details concerning the computational method used in this study, can be found elsewhere [4].

In accordance with the rapid nature of the transformation to the metastable structure, the mechanism must proceed through correlated atomic motions, and thus there must be a definite relationship between the metastable and initial wurtzite structures. In accordance with the suggestions by Sharma and Gupta [11], the lattice structures that were considered were those that could be linked to the wurtzite structure through a combination of a shifting of the alternately stacked basal planes of the wurtzite structure, associated with the softening of the $E_{2}$ phonon mode $[14,15]$, and deformation within the basal plane, associated with the softening of the effective elastic constant $C_{11}-C_{12}=2 C_{66}$ [16].

A systematic study of the total energy of face centered orthorhombic (fco) and face centered tetragonal (fct) structures were carried out. Based upon this systematic study, the most energetically favorable structure, and thus the most likely candidate for the metastable structure, is an fct structure shown in Fig. 4. As can be seen in Fig. 5, the total energy of this structure is only 0.05 eV/atom above the total energy of the final rocksalt structure. In comparison, the difference in energy for the fco structure proposed by Sharma and Gupta [11] as the metastable structure was found to be greater than $0.5 \mathrm{eV} /$ atom.

Also shown in Fig. 4 is the mechanism required to bring about the transformation from the wurtzite

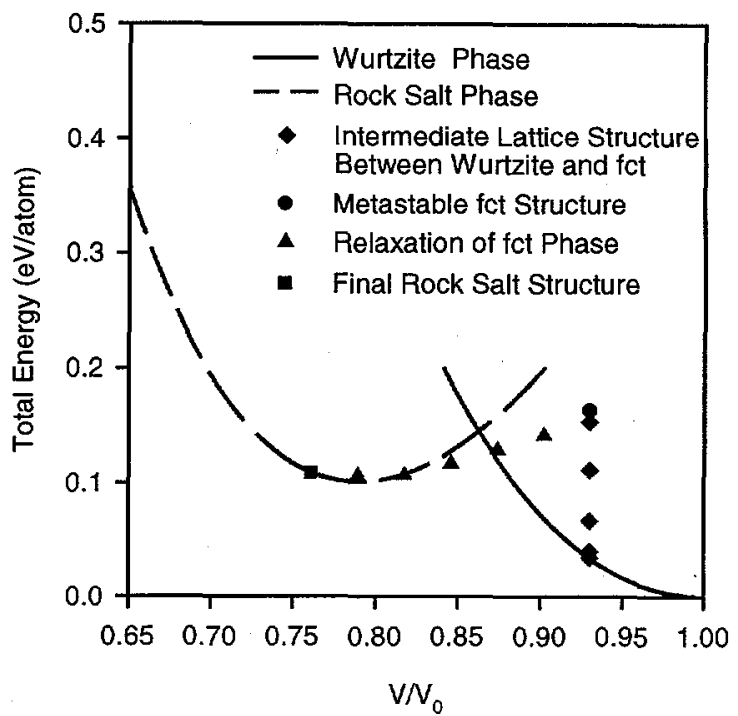

FIGURE 5. Comparison of the total energy of the wurtzite, fct, and rocksalt phases of CdS. Also shown are total energy calculations for continuous deformation between these phases.

structure to the proposed metastable fct structure. A combination of two deformations must occur. First, the alternately stacked basal planes, labeled $a$ and $b$, must shift out of phase with respect to each other by an amount such that the atoms marked 1 and 2 become collinear (designated by the arrows). This deformation is related to the $E_{2}$ phonon mode that softens with pressure $[14,15]$. Second, deformation within the basal planes must occur to bring about a transformation from the six-atom coordination of the hexagonal array to the four-atom coordination of the square array. This deformation is related to the effective elastic constant $C_{11}-C_{12}=2 C_{66}$ that also softens with pressure [16].

To determine if such deformation would require surmounting a large energy barrier, and thus refute this mechanism, total energy calculations were performed for a continuous deformation between the wurtzite and fct structures. The lattice parameters were systematically changed; with $A B$ increasing and $\mathrm{BC}$ decreasing in such a way that the volume remained constant, while the alternately stacked basal planes were systematically shifted with respect to each other such that the atoms marked 1 and 2 became collinear. The results of these calculations are shown in Fig. 5. It was found that the total energy of the lattice increased smoothly to that of the fct lattice, without having to 

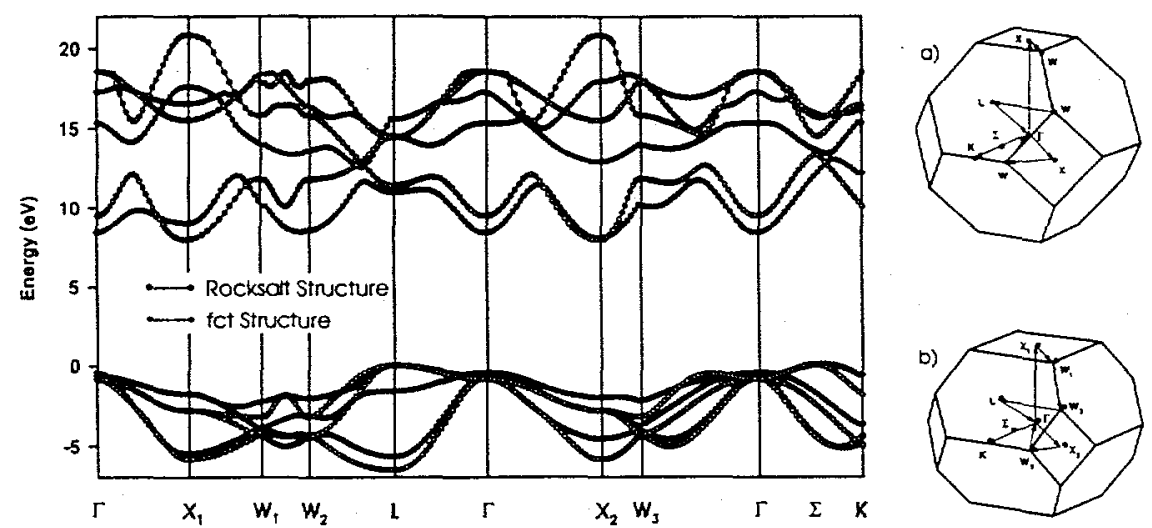

FIGURE 6. Comparison of the band structure of rocksalt and $\mathrm{fct} \mathrm{CdS}$ along certain symmetry directions of the Brillouin zone. These directions are shown in the Brillouin zones for the (a) rocksalt and (b) fct structures. Due to symmetry in the rocksalt structure, $X_{1}$ and $X_{2}$, and $W_{1}, W_{2}$, and $W_{3}$ are all the same symmetry points $X$ and $W$.

surmount an energy barrier. Also shown in Fig. 5 are total energy calculations for continuous deformation from the fct structure to the final rocksalt structure.

Based upon the total energy calculations, the fct structure is the most likely candidate for the metastable structure. To determine if this structure could be the structure of the material observed directly behind the shock front in the present experimental study, electronic band structure calculations were performed for the fct structure. The experimental results indicate that the electronic structure of the material directly behind the shock front is similar to that of the final rocksalt phase. Since Hartree-Fock methods characteristically overestimate the band gap energy of crystalline systems [17], electronic structure calculations were also performed for on the rocksalt structure to compare with the results obtained for the fct structure. Fig. 6 shows the results of these calculations. Also shown in the figure are the Brillouin zones for the two structures; the similarity in the Brillouin zones reflects the fact that the two crystal structures are very similar, as can be seen in Fig. 4.

The comparison shown in Fig. 6 indicates that both structures exhibit very similar band structures. An analysis of the density of states for these structures [4] reveals that the similarity in the basal planes for these structures accounts for the similarity in the band structures; the states involved in the transition are localized within the basal planes. Thus one would expect the band gap energy of the fct structure to be similar to that of the rocksalt structure, and further, one would expect the band gap energy to be fairly insensitive to the subsequent compression of the $\mathrm{c}$ axis in going from the fct structure to the final rocksalt structure.

\section{REFERENCES}

1. M.D. Knudson, Picosecond Electronic Spectroscopy to Understand the Shock-Induced Phase Transition in Cadmium Sulfide, Ph.D. Thesis, Washington State University, 1998 (unpublished)

2. M.D. Knudson and Y.M. Gupta, Phys. Rev. Lett. 81, 2938 (1998)

3. M.D. Knudson, K.A. Zimmerman, and Y.M. Gupta, Rev. Sci. Instrum. 70, 1743 (1999)

4. M.D. Knudson, Y.M. Gupta, and A.B. Kunz, Phys. Rev. B. 59, 11704 (1999)

5. J.I. Pankove, Optical Processes in Semiconductors, Prentice-Hall, New Jersey, 1971, p. 34-42

6. A.L. Edwards, T.E. Slykhouse, and H.G. Drickamer, J. Phys. Chem. Solids, 11, 140 (1959)

7. B. Batlogg, et al., Phys. Rev. B 27, 3920 (1983)

8. P. Cervantes, et al., Phys. Rev. B 54, 17585 (1996)

9. Y.M. Gupta, et al., Rev. Sci. Instrum. 51, 183 (1980)

10. Z.P. Tang and Y.M. Gupta, J. Appl. Phys. 81, 7203 (1997)

11. S.M. Sharma and Y.M. Gupta, Phys. Rev. B 58, 5964 (1998)

12. R. Dovesi, et al., CRYSTAL95 User Documentation, University of Toronto and Daresbury Laboratory, 1996

13. C. Lee, W. Yang, and R.G. Parr, Phys. Rev. B 37, 785 (1988)

14. S.S Mitra, et al., Phys. Rev. 186, 942 (1969)

15. R.J. Briggs and A.K. Ramdas, Phys. Rev. B 13. 5518 (1976)

16. J.A. Corll, Phys. Rev. 157, 623 (1967)

17. C. Pisani, R. Dovesi, and C. Roetti, Hartree-Fock AbInitio Treatment of Crystalline Systems, SpringerVerlag, Berlin, 1988 